\title{
Parental Presence during Induction reduced the time from patient entering operating room to the start of anesthetic induction
}

\author{
Takahiro Ando, M.D. ${ }^{1}$, Yuu Okamura, RN. ${ }^{1}$, Maki Goto, RN. ${ }^{1}$, Yoko Arakawa, M.D. ${ }^{1}$, Kimitoshi Nishiwaki, M.D.,Ph.D. ${ }^{2}$ \\ ${ }^{1}$ Nagoya University Hospital, Surgical section, Nagoya, Japan.
}

${ }^{2}$ Nagoya University Graduate School of Medicine, Dept of Anesthesiology , Nagoya, Japan.

\section{【Introduction】}

Hospital admissions and operations are stressful life events for patients. Furthermore, in pediatric patients, these events may have a major impact on their emotional response. It is natural for parents or guardians to feel anxiety and fear about anesthetizing and operating on their children. In order to assuage these anxieties and ease the emotions of children and their parents, our hospital started Parental Presence during Induction (PPI) for pediatric surgery in 2012. We evaluated the influence of PPI on the time from patient entering $O R$ to the start of anesthetic induction.

\section{【Procedure of PPI】}

From the ward to the entrance of the operating room (OR) area, the ward nurse and the child life specialist (CLS) move along with the patient plus his or her parents or guardians. From there, OR nurse joins in so they can all go together to the each OR. After entering the OR, the patient waits at the operation bedside. The surgeon and the anesthesiologist along with the OR nurse do the sign-in process with the presence of parents or guardians. After that, the patient monitoring devices are in place, taking off any clothes as necessary. Induction begins as the patient lies down or sits on the bed with the comfort of his or her parents or guardians. When the patient sleeps, the parents or guardians leave the OR along with the ward nurse or the CLS.

\section{【Subject and Method】}

From 2014 to 2016, A total of 342 pediatric patients (0-17 years old, ASA-PS1) undergoing inguinal hernia repair with general anesthesia. The time from patient entering OR to the start of anesthetic induction was retrospectively compared between in PPI group and non-PPI group.

【Results】

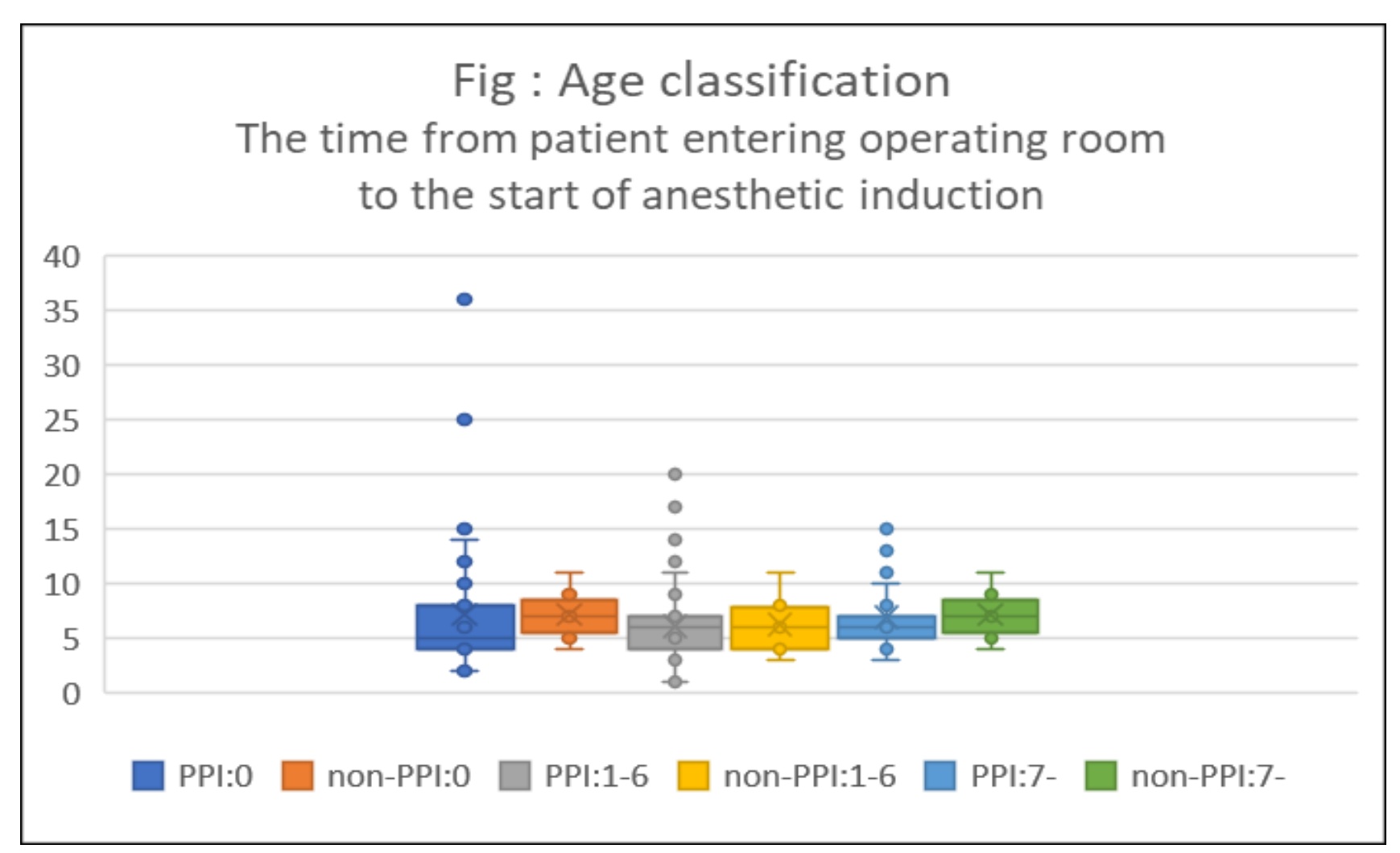

Table 1 : Year classification

\begin{tabular}{|c|c|c|c|c|c|}
\hline & Year & 2014 & 2015 & 2016 & total \\
\hline & $\mathrm{n}$ & 76 & 85 & 99 & 260 \\
\hline & non-PPI & 10 & 18 & 13 & 41 \\
\hline & $\min$ & 0 & 0 & 0 & 0 \\
\hline & $\max$ & 12 & 14 & 13 & 14 \\
\hline
\end{tabular}

\begin{tabular}{|c|c|c|c|c|c|c|}
\hline \multirow{3}{*}{ Age } & \multicolumn{2}{|c|}{ max } & 12 & 14 & 13 & 14 \\
\hline & \multirow{2}{*}{ mean $\pm S D$} & PPI & $3.7 \pm 2$. & $4.0 \pm 3.0$ & $4.1 \pm 3.2$ & $23.9 \pm 3.0$ \\
\hline & & non-PPI & $2.3 \pm 3$ & $5.3 \pm 5.0$ & $7.2 \pm 3.9$ & $95.2 \pm 4.6$ \\
\hline \multicolumn{3}{|c|}{$P$ value } & 0.18 & & 0.002 & 0.26 \\
\hline \multirow{4}{*}{$\begin{array}{l}\text { Time } \\
\text { (min) }\end{array}$} & \multicolumn{2}{|c|}{$\min$} & 1 & 1 & 2 & 1 \\
\hline & \multicolumn{2}{|c|}{$\max$} & 16 & 25 & 36 & 36 \\
\hline & \multirow{2}{*}{ mean $\pm S D$} & PPI & $5.5 \pm 2.5$ & $6.5 \pm 3.4$ & $6.9 \pm 4.2$ & $26.5 \pm 3.5$ \\
\hline & & non-PPI & $6.6 \pm 3.2$ & $7.8 \pm 2.1$ & $7.7 \pm 3.7$ & $77.6 \pm 3.3$ \\
\hline & $P$ valu & & 0.22 & 0.1 & 0.5 & 0.046 \\
\hline
\end{tabular}

Table 2 : Age classification

\begin{tabular}{|c|c|c|c|c|c|}
\hline & Age & 0 & $1-6$ & $7-$ & total \\
\hline & PPI & 49 & 159 & 52 & 260 \\
\hline & non-PPI & 9 & 16 & 16 & 41 \\
\hline & $\min$ & 2 & 1 & 3 & 1 \\
\hline Time & $\max$ & 36 & 20 & 16 & 36 \\
\hline
\end{tabular}

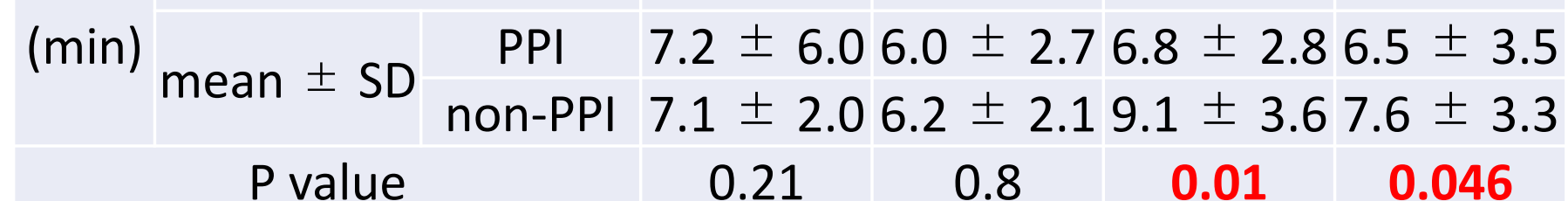
0.21
0.8
0.01
0.046

【Discussion】

In the group of 2016, it was suggested that the PPI performing rate decreased as age increased. In the total group, the time of PPI group showed shorter than that of non-PPI group. Although PPI feels like a troublesome thing to the anesthesiologists, PPI is effective to reduce the working time of Anesthesiologists. From the point of the working time, the age of children who PPI is particularly effective is over 7 years old. Because children over the age of 7 who have developed language ability and cognitive ability can understand what their parents say. In other age group, PPI group showed no significant difference of the time as compared with non-PPI group. So, it was suggested that PPI didn't increase workload of medical workers.

\section{【Conclusion】}

PPI reduces anxiety and fear of the patient about operation and anesthesia. As a result, the time from patient entering OR to the start of anesthetic induction was shortened and smooth, especially for over 7 years old group.

It was suggested that PPI is beneficial not only for burden but also for medical staff and patients. 